\title{
Aerodynamics of Axisymmetric Counterflowing Jets
}

\author{
Jaime Carpio ${ }^{\mathrm{a}}$, Amable Liñán ${ }^{\mathrm{b}}$, Antonio L. Sánchez ${ }^{\mathrm{c}}$, Forman A. Williams ${ }^{\mathrm{c}}$ \\ ${ }^{a}$ E. T. S. I. Industriales, Universidad Politécnica de Madrid, Madrid, 28006, Spain \\ ${ }^{b}$ E. T. S. I. Aeronáuticos, Pl. Cardenal Cisneros 3, 28040 Madrid, Spain \\ ${ }^{c}$ Dept. Mechanical and Aerospace Engineering, University of California San Diego, La Jolla CA \\ 92093-0411, USA
}

\begin{abstract}
The laminar flow resulting from impingement of two steadily fed low-Mach-number gaseous jets issuing into a stagnant atmosphere from coaxial cylindrical ducts at moderately large Reynolds numbers, often used in combustion experiments, is studied through numerical integrations of the Navier-Stokes equations. In the Reynolds-number range addressed, 501000, the flow of the approaching jets is nearly inviscid, with viscous effects and mixing being restricted to the thin mixing layers surrounding the jets and to a thin layer located at the separating stream surface. The analysis of the main inviscid flow shows that only two parameters, based on the scales associated with the radius and the velocity profiles of the two feed streams, are needed to characterize the flow, namely, the ratio of the inter-jet separation distance to the duct radius and the ratio of momentum fluxes of the jets. The numerical results for uniform and Poiseuille velocity profiles provide, in particular, the value of the strain rate at the stagnation point for use in the analysis of experimental studies of counterflow premixed and diffusion flames.
\end{abstract}

Keywords: counterflow combustor, strain rate, impinging jets

\section{Introduction}

Counterflow burners have been widely used by the combustion community for over fifty years in investigations of premixed, partially premixed, and non-premixed flames [1, 2]. Slot-jet counterflow combustors producing planar flows are employed by some researchers [3], but most experimental arrangements involve axisymmetric flow, addressed in the present paper. Although the computations pertain to an apparatus employing screen-free nozzles and ducts, as sketched in Fig. 1, much of the following discussion also applies to other experimental setups, such as opposing flows through porous plates of honeycombs or screens that provide significant flow resistance. In the figure, two opposed steady round jets issue into a stagnant atmosphere from aligned nozzles of the same radius $R$ separated a distance $2 H$. Although perfectly symmetric configurations involving identical jets are of interest, for example in twin-flame studies, in many situations the properties of the two streams, identified by subscripts 1 and 2 , are different, with different densities $\rho_{1}$ and $\rho_{2}$, viscosities $\mu_{1}$ and $\mu_{2}$, and volume fluxes $Q_{1}$ and $Q_{2}$. The mean jet velocity $U_{m}=\left[Q_{1} /\left(\pi R^{2}\right)\right] \sim$ $\left[Q_{2} /\left(\pi R^{2}\right)\right]$ used in experiments is typically much lower than the sound speed, resulting in two constant-density jets having spatial variations of pressure small compared with the 
ambient pressure, and such that $U_{m}^{2} \gg g R$ to minimize buoyancy effects, not considered here. In addition, in laminar combustion experiments the typical values of the relevant Reynolds number $R e=\rho_{1} Q_{1} /\left(\pi R \mu_{1}\right) \sim \rho_{2} Q_{2} /\left(\pi R \mu_{2}\right)$ range from about a hundred to about a thousand, large enough that the flow in the approaching streams is nearly inviscid. Mixing and viscous effects are confined to layers, of small thickness $R / R e^{1 / 2} \ll 1$ [4], one localized between the two opposing streams and the others at the two fluid surfaces originating at the rim of the ducts. This justifies the use of the Euler equations outside the mixing layer.

This description is simplified in the central region near the stagnation point, where the flow is self-similar both outside and inside the mixing layer. There the radial velocity grows linearly with the radial distance $r^{\prime}$ in the form $A_{1} r^{\prime} / 2$ in the inviscid stream 1 and $A_{2} r^{\prime} / 2$ in the inviscid stream 2. The strain rates $A_{1}$ and $A_{2}$, both of order $U_{m} / R$, are related by the condition of equal pressure across the mixing layer, so that $\rho_{2} A_{2}^{2}=\rho_{1} A_{1}^{2}$. The flow in the reactive mixing layer in this near-stagnation-point region is also self-similar, with the temperature and composition varying with the distance to the stagnation plane, leading to a one-dimensional problem [5], amenable to numerical integration by standard commercial codes (e.g. [6]), and the resulting one-dimensional counterflow model has been the basis for studies of flame-flow interactions in nonpremixed and premixed combustion [7]. At leading order in the limit $R e \gg 1$, the flame structure in the vicinity of the axis depends on the outer flow only through the inviscid value of the strain rate $A_{1}=A_{2} \sqrt{\rho_{2} / \rho_{1}}$, which can be determined in the first approximation from chemically frozen experimental measurements [8] or computations $[9,10]$. In the presence of combustion the resulting relative variations of $A_{1}$ and $A_{2}$ are small, of order $R e^{-1 / 2}$, associated with the outer-flow displacements induced in the mixing layer [4].

Previous numerical calculations of laminar axisymmetric flow fields for chemically frozen impinging jets have considered either two identical jets $[9,11]$ or the closely related problem of a jet impinging on flat wall [10, 12-17]. For the latter flow, the complete Navier-Stokes equations have been employed to address Reynolds-number dependences [10, 15], verifying that for moderately large $R e$ the flow structure approaches the inviscid solution [10], which can be computed with a stream-function/vorticity formulation [10, 12-14, 16, 17]. The present numerical integrations of the Navier-Stokes equations, selected results of which are shown on the right-hand side of Fig. 1, extend previous results to two jets that are not identical, with the limit $R e \gg 1$ also addressed to reveal the minimum set of nondimensional parameters determining the flow.

\section{Problem formulation}

Referring to Fig. 1 the resulting axisymmetric flow will be described using cylindrical coordinates centered at the middle point along the axis. We use the equal radius $R$ of the feed streams and the mean feed velocity $U_{m}=Q_{1} /\left(\pi R^{2}\right)$ of stream 1 , together with $R / U_{m}$, and $\rho_{1} U_{m}^{2}$, as characteristic scales for length, velocity, time, and pressure differences from the external ambient pressure, resulting in the dimensionless coordinates $\mathbf{x}=(x, r)$, velocity $\mathbf{v}=\left(v_{x}, v_{r}\right)$, time $t$, and pressure difference $p^{\prime}(x, r)$. Correspondingly, the thermodynamic and transport properties of stream 1 will be used to define a dimensionless density $\rho$, temperature $T$, viscosity $\mu$, heat conductivity $k$, and molecular diffusivity $D_{i}$ of species $i$. Buoyancy forces are neglected in the analysis, an appropriate simplification for small combustors with 
sufficiently large Froude numbers $U_{m}^{2} /(g R) \gg 1$. For the low-Mach-number conditions of most combustion applications, the conservation equations [18], written in terms of the dimensionless variables defined above, reduce to

$$
\begin{aligned}
\frac{\partial \rho}{\partial t}+\nabla \cdot(\rho \mathbf{v}) & =0 \\
\rho \frac{\partial \mathbf{v}}{\partial t}+\rho \mathbf{v} \cdot \nabla \mathbf{v} & =-\nabla p^{\prime}+\frac{1}{R e} \nabla \cdot \tau \\
\rho \frac{\partial T}{\partial t}+\rho \mathbf{v} \cdot \nabla T & =\frac{1}{\operatorname{Pr} R e} \nabla \cdot(k \nabla T), \\
\rho \frac{\partial Y_{i}}{\partial t}+\rho \mathbf{v} \cdot \nabla Y_{i} & =\frac{1}{S c_{i} R e} \nabla \cdot\left(\rho D_{i} \nabla Y_{i}\right) .
\end{aligned}
$$

Here, $\tau=\mu\left(\nabla \mathbf{v}+\nabla \mathbf{v}^{T}\right)$ is the viscous stress tensor, $R e=\rho_{1} Q_{1} /\left(\pi R \mu_{1}\right)$ is the relevant Reynolds number based on the viscosity in the reference feed stream 1, and $\operatorname{Pr}$ and $S c_{i}$ are the Prandtl number of the gas and the Schmidt number of species $i$, assumed to be constant. The above equations, supplemented with expressions for the temperature and composition dependences of the dimensionless transport coefficients $k, \mu$, and $D_{i}$ and with the equation of state written in the low-Mach-number form

$$
\rho T \sum_{i}\left(Y_{i} / W_{i}\right)=1
$$

with $W_{i}$ denoting the molecular mass of species $i$ scaled with the mean molecular mass of stream 1, must be integrated with appropriate boundary conditions, including a nonslip condition for the velocity at the walls (i.e. $\mathbf{v}=0$ at $r=1$ for $H / R \leq|x|<\infty$ ) and known boundary density and velocity distributions upstream from the nozzle exit, such as

$$
\left\{\begin{array}{lll}
\rho=1, & v_{x}=U_{1}(r) & \text { as } x \rightarrow-\infty \\
\rho=\rho_{2} / \rho_{1}, & v_{x}=-\left(Q_{2} / Q_{1}\right) U_{2}(r) & \text { as } x \rightarrow \infty
\end{array}\right.
$$

for $0 \leq r \leq 1$, where $U_{1}(r)$ and $U_{2}(r)$ represent nondimensional velocities of the feed streams satisfying $2 \int_{0}^{1} U_{1} r \mathrm{~d} r=2 \int_{0}^{1} U_{2} r \mathrm{~d} r=1$ (i.e. $U_{1}=U_{2}=1$ and $U_{1}=U_{2}=2\left(1-r^{2}\right)$ for uniform flow and for Poiseuille flow, respectively). These velocity distributions are imposed in the numerical calculations at a distance of the order of, although moderately larger than, $R$ upstream from the duct exits, outside the region where the flow in the ducts is affected by upstream perturbations coming from the collision region.

Besides the Prandtl number and Schmidt numbers, the problem depends on four parameters, namely, the geometric parameter $H / R$, the Reynolds number $R e$, and the density and volume-flux ratios $\rho_{2} / \rho_{1}$ and $Q_{2} / Q_{1}$. The dependence can be reduced by noting that the Reynolds number Re is moderately large in typical applications, so that the flow of the counterflowing streams is nearly inviscid, with significant effects of molecular diffusion occurring only near the jet boundaries and in the separating mixing layer.

\subsection{Inviscid steady flow}

For steady flow in the limit $R e \gg 1$, equations (3) and (4) reduce to $\mathbf{v} \cdot \nabla T=0$ and $\mathbf{v} \cdot \nabla Y_{i}=0$, respectively, revealing that the temperature and composition, and therefore 
the density according to (5), remain constant along the streamlines, thereby reducing (1) to $\nabla \cdot \mathbf{v}=0$. The description of the resulting solenoidal velocity field can be simplified by introducing the stream function $\psi$, derived from the steady mass conservation equation with constant density in each of the counterflowing streams, so that

$$
v_{x}=\frac{1}{r} \frac{\partial \psi}{\partial r} \quad \text { and } \quad v_{r}=-\frac{1}{r} \frac{\partial \psi}{\partial x}
$$

For this axisymmetric flow the vorticity is azimuthal, with magnitude

$$
\omega_{\theta}=\frac{\partial v_{r}}{\partial x}-\frac{\partial v_{x}}{\partial r}
$$

given in terms of the stream function by

$$
-r \omega_{\theta}=\frac{\partial^{2} \psi}{\partial x^{2}}+r \frac{\partial}{\partial r}\left(\frac{1}{r} \frac{\partial \psi}{\partial r}\right) .
$$

Since the flow is inviscid and steady, both the stagnation pressure $p^{\prime}+\rho \mathbf{v}^{2} / 2$ and the ratio $\omega_{\theta} / r$ remain constant along any given streamline [19], so that

$$
p^{\prime}+\frac{1}{2} \rho|\nabla \psi|^{2} / r^{2}=H(\psi)
$$

and

$$
\omega_{\theta} / r=\Omega(\psi) .
$$

Equation (10) can be evaluated along the axis to provide the overpressure $p_{o}^{\prime}$ at the stagnation point, which must be equal for both streams, so that

$$
p_{o}^{\prime}=p_{1}^{\prime}+\frac{1}{2} U_{1}^{2}(0)=p_{2}^{\prime}+\frac{1}{2} \Lambda^{2} U_{2}^{2}(0),
$$

where $p_{1}^{\prime}$ and $p_{2}^{\prime}$ are the overpressures in the feed streams, with the parameter

$$
\Lambda^{2}=\frac{\rho_{2}}{\rho_{1}}\left(\frac{Q_{2}}{Q_{1}}\right)^{2}
$$

measuring the ratio of the momentum fluxes of the two jets. The function $\Omega(\psi)$ can be evaluated by using the boundary distributions in the feed streams

$$
\begin{array}{r}
\Omega=-\frac{1}{r} \frac{\mathrm{d} U_{1}}{\mathrm{~d} r}, \quad \psi=\int_{0}^{r} U_{1} r \mathrm{~d} r, \\
\Omega=\left(\frac{Q_{2}}{Q_{1}}\right) \frac{1}{r} \frac{\mathrm{d} U_{2}}{\mathrm{~d} r}, \quad \psi=-\left(\frac{Q_{2}}{Q_{1}}\right) \int_{0}^{r} U_{2} r \mathrm{~d} r,
\end{array}
$$

associated with (6). In particular, $\Omega=0$ for uniform flow, while for Poiseuille flow $\Omega$ is constant in both streams; $\Omega=4$ for $0 \leq \psi \leq 1 / 2$, and $\Omega=-4\left(Q_{2} / Q_{1}\right)$ for $-\frac{1}{2}\left(Q_{2} / Q_{1}\right) \leq$ $\psi \leq 0$.

The problem reduces to the integration of

$$
\frac{\partial^{2} \psi}{\partial x^{2}}+r \frac{\partial}{\partial r}\left(\frac{1}{r} \frac{\partial \psi}{\partial r}\right)=-r^{2} \Omega(\psi)
$$


obtained by using (11) in (9). In the feed streams the stream function is given for $0 \leq r \leq 1$ by $\psi=\int_{0}^{r} U_{1} r \mathrm{~d} r$ as $x \rightarrow-\infty$ and by $\psi=-\left(Q_{2} / Q_{1}\right) \int_{0}^{r} U_{2} r \mathrm{~d} r$ as $x \rightarrow \infty$. The condition $\psi=0$ applies at $r=0$ for $-\infty<x<+\infty$, while $\psi=1 / 2$ at $r=1$ for $-\infty<x \leq-H / R$ and $\psi=-\frac{1}{2}\left(Q_{2} / Q_{1}\right)$ at $r=1$ for $H / R \leq x<+\infty$. On the jet free boundaries $r_{1}(x)$ and $r_{2}(x)$ separating the jet flow from the outer stagnant gas, corresponding to $\psi=-1 / 2$ and $\psi=-\frac{1}{2}\left(Q_{2} / Q_{1}\right)$, the condition of constant pressure can be written from (10) and (12) in the form

$$
\begin{aligned}
& \frac{1}{2} \frac{|\nabla \psi|^{2}}{r^{2}}=p_{o}^{\prime}-\frac{1}{2}\left[U_{1}^{2}(0)-U_{1}^{2}(1)\right] \quad \text { at } \quad r=r_{1}(x) \\
& \frac{1}{2}\left(\frac{\rho_{2}}{\rho_{1}}\right) \frac{|\nabla \psi|^{2}}{r^{2}}=p_{o}^{\prime}-\frac{1}{2} \Lambda^{2}\left[U_{2}^{2}(0)-U_{2}^{2}(1)\right] \quad \text { at } \quad r=r_{2}(x)
\end{aligned}
$$

involving the unknown overpressure $p_{o}^{\prime}$ at the stagnation point, whose location $x=x_{o}$ along the axis is to be determined as part of the solution. The stream surface $\psi=0$ originating at the stagnation point, which separates the two opposing fluids, is an additional unknown surface $x=x_{s}(r)$, to be determined from the condition of equal pressure on both opposing flows

$$
|\nabla \psi|_{-}^{2}=\left(\frac{\rho_{2}}{\rho_{1}}\right)|\nabla \psi|_{+}^{2}
$$

where the subscripts - and + denote the derivatives at $\psi=0^{-}$and $\psi=0^{+}$, respectively.

\subsection{Reduced formulation}

The problem can be simplified considerably, removing the need to consider specifically the separating surface and reducing the parametric dependence, by introducing alternative functions $\hat{\psi}$ and $\hat{\Omega}$ defined by $\hat{\psi}=\psi$ and $\hat{\Omega}=\Omega$ for $\psi>0$ and by $\hat{\psi}=\left(\rho_{2} / \rho_{1}\right)^{1 / 2} \psi$ and $\hat{\Omega}=\left(\rho_{2} / \rho_{1}\right)^{1 / 2} \Omega$ for $\psi<0$. Unlike $|\nabla \psi|$, the gradient of $\hat{\psi}$ remains continuous across the separating surface $\hat{\psi}=0$, as can be inferred from (19), and the vorticity equation (16) reduces to

$$
\frac{\partial^{2} \hat{\psi}}{\partial x^{2}}+r \frac{\partial}{\partial r}\left(\frac{1}{r} \frac{\partial \hat{\psi}}{\partial r}\right)=-r^{2} \hat{\Omega}(\hat{\psi})
$$

which simplifies for uniform feed velocities, leading to $\hat{\Omega}=0$, and for Poiseuille flow, leading to $\hat{\Omega}=4$ for $0 \leq \hat{\psi} \leq 1 / 2$, and $\hat{\Omega}=-4 \Lambda$ for $-\Lambda / 2 \leq \psi \leq 0$. The boundary conditions for integration include

$$
\left\{\begin{array}{lll}
\hat{\psi}=0 & \text { at } r=0 & \text { for }-\infty<x<+\infty \\
\hat{\psi}=1 / 2 & \text { at } r=1 & \text { for }-\infty<x \leq-H / R \\
\hat{\psi}=-\Lambda / 2 & \text { at } r=1 & \text { for } H / R \leq x<+\infty
\end{array}\right.
$$

along with

$$
\begin{gathered}
\hat{\psi}=1 / 2 ; \quad \frac{1|\nabla \hat{\psi}|^{2}}{2}=p_{o}^{\prime}-\frac{1}{2}\left[U_{1}^{2}(0)-U_{1}^{2}(1)\right] \quad \text { at } \quad r=r_{1}(x) \\
\hat{\psi}=-\Lambda / 2 ; \quad \frac{1}{2} \frac{|\nabla \hat{\psi}|^{2}}{r^{2}}=p_{o}^{\prime}-\frac{1}{2} \Lambda^{2}\left[U_{2}^{2}(0)-U_{2}^{2}(1)\right] \quad \text { at } \quad r=r_{2}(x)
\end{gathered}
$$


at the free surfaces separating the jets from the stagnant air and the boundary velocity distributions

$$
\begin{aligned}
& \hat{\Omega}=-\frac{1}{r} \frac{\mathrm{d} U_{1}}{\mathrm{~d} r}, \quad \hat{\psi}=\int_{0}^{r} U_{1} r \mathrm{~d} r \quad \text { as } x \rightarrow-\infty, \\
& \hat{\Omega}=\frac{\Lambda}{r} \frac{\mathrm{d} U_{2}}{\mathrm{~d} r}, \quad \hat{\psi}=-\Lambda \int_{0}^{r} U_{2} r \mathrm{~d} r \quad \text { as } x \rightarrow \infty,
\end{aligned}
$$

for $0 \leq r \leq 1$. This last equation provides an implicit representation for the function $\hat{\Omega}(\hat{\psi})$, needed to integrate $(20)$.

The integration would determine, in particular, the axial location $x_{o}$ of the stagnation point, along with the associated local stream-function description

$$
\hat{\psi}=-A r^{2}\left[x-x_{s}(r)\right] / 2,
$$

where $A=A_{1} /\left[Q_{1} /\left(\pi R^{3}\right)\right]$ is the nondimensional value of the strain rate at the stagnation point, and $x_{s}(r)$ is the surface separating both streams, given by

$$
x_{s}=x_{o}+\frac{r^{2}}{2 r_{c}}
$$

near the stagnation point, where $r_{c}$ is the local radius of curvature. While this curvature contributes a term of order $r^{4}$ to the expansion of the stream function about $r=0$ in (26), there also is a contribution of order $r^{4}$ proportional to $\left(x-x_{o}\right)$, so that, through terms of order $r^{3}$, the corresponding nondimensional radial velocity is

$$
v_{r}=A r / 2+B r^{3} / 8,
$$

the pressure decreasing locally with increasing $r$ in proportion to $(A r / 2)^{2}$ at the axis. The sensitivities of the values of $x_{o}, r_{c}, A$, and $B$ to the shapes of the velocity profiles in the approach flow are of interest.

The formulation given in (20)-(25) is attractive for two reasons. First of all, it removes the need to consider the separating surface $\hat{\psi}=0$ as a free surface in numerical integrations of the inviscid flow. Secondly, it demonstrates that the low-Mach-number flow induced by opposed gas jets at moderately large Reynolds numbers, found in counterflow burners, depends only on the shapes of the velocity profiles $U_{1}(r)$ and $U_{2}(r)$ in the feed streams and on the two parameters $H / R$ and $\Lambda=\left(\rho_{2} / \rho_{1}\right)^{1 / 2}\left(Q_{2} / Q_{1}\right)$, the latter effectively embodying the dependences on density and velocity ratio.

\section{Results of numerical integrations of the Navier-Stokes equations}

The reduced parametric dependence identified above was considered in defining the conditions for integration of the Navier-Stokes equations (1)-(4) for values of the Reynolds number in the range $50 \leq R e \leq 1000$. In particular, according to the previous reasoning, under the large-Reynolds-number conditions considered here the temperature and composition of the feed streams enter in the outer nearly inviscid solution only through the factor $\left(\rho_{2} / \rho_{1}\right)^{1 / 2}$ in 
$\Lambda=\left(\rho_{2} / \rho_{1}\right)^{1 / 2}\left(Q_{2} / Q_{1}\right)$, so that solutions with different composition and different feed temperatures but equal values of $\Lambda$ exhibit small relative differences of order $R e^{-1 / 2}$ outside the mixing layers bounding the two jet streams. For simplicity, the integrations below pertain to isothermal jets of two different gases of mass fractions $Y_{1}$ and $Y_{2}$ with a Schmidt number of $S c=0.7$, appropriate for air, and corresponding boundary conditions in the feed streams away from the pipe exit given by $Y_{1}-1=Y_{2}=0$ as $x \rightarrow-\infty$ and $Y_{1}=Y_{2}-1=0$ as $x \rightarrow \infty$. The density of the ambient gas, also with the same Schmidt number, is taken to be equal to that of jet 1 , thereby reducing the equation of state (5) to $\rho\left[1-Y_{2}+Y_{2} /\left(\rho_{2} / \rho_{1}\right)\right]=1$, which can be used in (4) to give

$$
\frac{\partial \rho}{\partial t}+\mathbf{v} \cdot \nabla \rho=\frac{\rho}{S c R e} \nabla \cdot\left(\rho^{-1} \nabla \rho\right)
$$

as a conservation equation for the density. The numerical integrations of (1), (2), and (29) employed a cylindrical domain of radius $r=5$ extending axially between $x=-4$ and $x=$ 4. Constant pressure was imposed at the ambient-gas boundaries, although computations with external co-flows, at velocities less than maximum jet velocities, to delay instabilities, exerted no observable centerline effects, supporting assumptions of negligible influences of experimental protective blanket streams. A nonslip condition $\mathbf{v}=0$ was employed at the pipe wall in integrations with Poiseuille flow in the feed streams. For uniform flow, however, a slip-flow condition $v_{r}=0$ at the wall was used instead, to make the results of the integrations independent of the axial extent of the integration domain, thereby providing results for an opposite limiting case, with flows in other configurations, such as nozzle-shaped jets, tending to fall between these two limits.

The geometrical parameter $H / R$ was varied in the range $0.1 \leq H / R \leq 2.0$ representative of counterflow-flame experiments. Opposed-jet configurations involving identical jets with larger values of $H / R$, of interest in industrial applications, are known to be prone to oscillatory instabilities [20], resulting in the mixing layer becoming attached to one or the other jet, an aspect of the problem not investigated here. Different values of the density ratio $\rho_{2} / \rho_{1}$ and of the volume-flux ratio $Q_{2} / Q_{1}$ were computed, with resulting values of $\Lambda=\left(\rho_{2} / \rho_{1}\right)^{1 / 2}\left(Q_{2} / Q_{1}\right)$ varying from $\Lambda=1$ to $\Lambda=2$. Results of integrations, obtained with use made of a previously developed numerical code [21], are summarized in Figs. 1-4.

The general flow structure is shown on the right-hand side of Fig. 1, which includes two snapshots for isopycnic flow with $R e=1000$ and $H / R=1$. Isocontours of mass fraction are used to reveal the boundaries of the shear layers bounding the jets and of the separating mixing layer. It is seen from this figure that, at this Reynolds number, the mixing layer occupies a very small fraction of the flow field, although this fraction will increase as a result of displacement effects through the density decrease associated with the heat release when there is a flame in the boundary layer [4]. Besides the symmetric case $Q_{2} / Q_{1}=1$, the case $Q_{2} / Q_{1}=2$ is used in this figure to illustrate the mixing-layer displacement and the jet deflection occurring for configurations with unbalanced momentum flux. For this relatively large Reynolds number the external bounding surfaces are seen to be affected by Kelvin-Helmhotz instabilities that preclude the establishment of fully steady conditions in the radial jet, but it is noteworthy that, despite these instabilities, the separating mixing layer remains virtually unperturbed and displays in the vicinity of the axis a steady onedimensional structure with parallel isosurfaces. 
The shape of the separating streamline $x=x_{s}(r)$ that originates at the stagnation point $(x, r)=\left(x_{o}, 0\right)$ is investigated in Fig. 2 for configurations with unbalanced momentum flux, variations of the momentum-flux ratio $\Lambda$ being imposed computationally by varying the volume-flux ratio $Q_{2} / Q_{1}$. For $\Lambda=1$, the separating streamline is the plane $x_{s}(r)=0$, by symmetry. Since the streamline $x=x_{s}(r)$ obtained with a given value of $\Lambda$ and that obtained with its reciprocal $1 / \Lambda$ are mirror images of each other about the plane $x=0$, only results for $\Lambda>1$ are considered in the figure. Besides separating streamlines for both uniform and Poiseuille velocity profiles with $H / R=1$, the figure includes the dependence on $H / R$ of the stagnation-point location $x_{o}$ and of the curvature $1 / r_{c}$ defining in (27) the local shape of $x=x_{s}(r)$ near the axis. Clearly, for the symmetric case $\Lambda=1$ these two quantities are simply $x_{o}=1 / r_{c}=0$ irrespective of the value of $H / R$. As can be seen, the unbalanced momentum flux results in a displacement of the stagnation point towards the jet with lower momentum, giving relative displacements that increase with increasing values of $\Lambda$ and $H / R$. The separating streamline remains relatively flat near the stagnation point, with values of the local dimensionless curvature $1 / r_{c}$ that remain below 0.5 in the cases considered. The comparison between the results with uniform and Poiseuille velocity profiles indicates that the former lead to smaller stagnation-point displacements and flatter separating streamlines. In addition, the value of $B$ in (28) (not shown) has opposite signs for the uniform and parabolic inlet profiles, being positive in the former case but negative in the latter, as might be expected from the shapes of these profiles.

The flow structure near the stagnation point is further investigated in Fig. 3 by plotting the distributions of $v_{x}(x, 0)$ and $v_{r}(0, r)$ in the symmetric case $H / R=1, \rho_{2} / \rho_{1}=1, Q_{2} / Q_{1}=$ 1 , and $U_{1}=U_{2}=1$ for $R e=(50,250,1000)$. The axial and radial velocity components display near the stagnation point the expected linear dependence $v_{x}=-A x$ and $v_{r}=$ $A r / 2$ corresponding to the potential-flow stagnation-point solution. In the right-hand figure, for $R e=1000$ the Kelvin-Helmhotz instabilities of the outer shear layers cause the radial velocity to be unsteady at large radial distances, the profile shown being a representative one, although the solution remains steady, nearly unperturbed, in the near-stagnation-point region, as previously indicated.

The results reveal that the linear variation of the axial and radial velocity components with the distance from the stagnation point applies in a fairly large region. The linear variation of the radial velocity is associated with a constant value of the reduced pressure gradient $-(1 / r) \partial p^{\prime} / \partial r=A^{2} / 4$ at the separating interface, as is needed to ensure the validity of the one-dimensional solution for the mixing layer [5]. Although these one-dimensional solutions, applicable at all Reynolds numbers, exhibit axial variations of $\partial v_{r} / \partial r$ that are different for codes with different boundary conditions, all such variations occur within the very thin mixing layers seen in Fig. 1, the present values of $\partial v_{r} / \partial r=A / 2$ and $\partial v_{r} / \partial r=$ $\left(\rho_{1} / \rho_{2}\right)^{1 / 2} A / 2$ in the two streams essentially being those just outside the mixing layer. The departures from this linear variation, measured by the last term in (28), are seen in Fig. 3 to be relatively small, so that the mixing layer can be expected to maintain a one-dimensional structure up to distances of about half a pipe radius, as was found for all of the cases investigated. The computations revealed that, while for the case $U_{1}=U_{2}=1$ shown in Fig. 3 the resulting radial velocity near the stagnation point is larger than $v_{r}=A r / 2$, corresponding to positive values of $B$ in (28), the opposite is found for Poiseuille flow in the feed streams. The negative value of $\mathrm{B}$ for the parabolic inlet profile exhibits a magnitude 

little on $\Lambda$ ) is practically independent of $H / R$ but increases substantially with increasing $\Lambda$, reaching beyond -20 at $\Lambda=2$. This behavior is consistent with the reduction in the effective flow restriction with increasing radius, associated with the Poiseuille proflie, and the increase in the magnitude with increasing $\Lambda$ is a consequence of the decrease of the relative momentum of stream 1 . These differences emphasize the very noticeable effects of the two extreme inlet-profiles selected; in intermediate cases, such as those of nozzletype counterflows, the initial departure from linearity in the right-hand panel may be either positive or negative, depending on the specific design of the experiment. This is the largest influence of the experimental configuration found in the present study.

The velocity profiles shown in Fig. 3 indicate that the stagnation-point strain rate, nondimensionalized only on the basis of stream $1, A=A_{1} /\left[Q_{1} /\left(\pi R^{3}\right)\right]$ is very similar for the three Reynolds numbers, with noticeable differences appearing only at the lowest Reynolds number. This is quantitatively investigated in the upper inset of Fig. 3, which gives the corresponding value of $A$ obtained for different $H / R$ and $R e=(50,250,1000)$ with $\rho_{2} / \rho_{1}=1$, $Q_{2} / Q_{1}=1$, and $U_{1}=U_{2}=1$. The resulting differences in strain rate are seen to become smaller as the Reynolds number increases, consistent with the predicted order of magnitude $R e^{-1 / 2}$ of the departures from the inviscid limit. Viscous effects are more significant for larger internozzle distances, when the growth of the bounding shear layers downstream from the jet exit influences the resulting impingement region. It is of interest that the differences in $A$ between the cases $R e=250$ and $R e=1000$ remain smaller than $5 \%$ over the whole range of values of $H / R$ considered.

According to the reduced inviscid formulation introduced earlier the value of the strain rate $A_{1}=A_{2} \sqrt{\rho_{2} / \rho_{1}}$ expressed in the dimensionless form $A=A_{1}\left[Q_{1} /\left(\pi R^{3}\right)\right]$ depends on $\rho_{2} / \rho_{1}$ and $Q_{2} / Q_{1}$ through the single parameter $\Lambda=\left(\rho_{2} / \rho_{1}\right)^{1 / 2}\left(Q_{2} / Q_{1}\right)$, so that results with different density and different volume-flux ratios but identical values of $\Lambda$ should give the same dimensionless strain rate. This is tested in the lower inset in Fig. 3, which compares the axial velocity distribution $v_{x}(x, 0)$ obtained for $R e=1000$ with $\rho_{2} / \rho_{1}=1$ and $Q_{2} / Q_{1}=1$ to that obtained with $\rho_{2} / \rho_{1}=4$ and $Q_{2} / Q_{1}=0.5$, both cases having $\Lambda=1$. For $\rho_{2} / \rho_{1}=4$ the jump in density across the mixing layer results in a jump in velocity gradient, in agreement with the condition $A_{1}=A_{2} \sqrt{\rho_{2} / \rho_{1}}$. The plot also shows that the velocity gradients to the left of the stagnation point for the two computations $\rho_{2} / \rho_{1}=1$ and $\rho_{2} / \rho_{1}=4$, corresponding to the values of $A=A_{1}\left[Q_{1} /\left(\pi R^{3}\right)\right]$, are indistinguishable, in agreement with the previous predictions. Furthermore, when the axial velocity $v_{x}(x, 0)$ obtained for $\rho_{2} / \rho_{1}=4$ is scaled with $\rho^{1 / 2}$, giving the distribution represented with the empty circles in the inset, the resulting curve falls on top of the velocity distribution of the uniform-density case, also in agreement with the inviscid results.

Fig. 4 is a correlation of all of the numerical resuts obtained at high Reynolds numbers for the strain rates right outside the mixing layer, which is the quantity of interest in combustion. These plots, which extend over a wide range of $H / R$ that encompasses essentially all combustion applications, reveal excellent scaling with

$$
\frac{A}{\Lambda^{1 / 2}}=\frac{\left(A_{1} A_{2}\right)^{1 / 2}}{\left(Q_{1} Q_{2}\right)^{1 / 2} /\left(\pi R^{3}\right)},
$$

which shows that the geometric mean of the strain rate across the mixing layer scales with 
the geometric mean of the volume fluxes. When there is interest in $A$, the strain rate on one side of the layer, nondimensionalized by the time scale constructed from the volume flow rate on that side, then it may be assumed to increase with the momentum-flux ratio in proportion to its square root. The results obtained with Poiseuille velocity profiles in the feed streams are particularly simple, in that $A / \Lambda^{1 / 2} \simeq 4.5$ for all three values of $\Lambda$ in the whole range $0.1 \leq H / R \leq 2$ explored in the computations. For the limit of a uniform velocity profile, on the other hand, not only is the value of $A / \Lambda^{1 / 2}$ less than half that for Poiseuille, but also the slow decrease with increasing $H / R$ is more pronounced. The shape of the velocity profile exerts the greatest influence on this strain rate. For the two limiting cases addressed, the curves in Fig. 4 together with the condition $A_{1}=A_{2} \sqrt{\rho_{2} / \rho_{1}}$ enable evaluation of the strain rates in counterflow burners involving opposed round jets issuing from screen-free nozzles provided that the associated jet Reynolds numbers are sufficiently large.

\section{Conclusions}

Computations have now produced scaling relations for flow fields and strain rates in axisymmetric counterflows that extend to conditions in which the momentum fluxes of the two streams are not balanced and that include the full range of ratios of separation distances to duct radii. Although the relevance of the ratio of momentum fluxes of the two streams is consistent with the inviscid formulation for large Reynolds numbers presented here, the virtual independence of the strain rate just outside the mixing layer from the nondimensional interjet distance, as well as its geometric-mean scaling (30), are computational results that are not immediate consequences of that formulation. Of the two physical dimensions involved, $R$ and $H$ of Fig. 1, the stagnation-point strain rate increases as the former decreases but remains practically independent of the latter, as might be expected from the observations that, as the ratio $H / R$ approaches zero the external inviscid flow approaches that from ring source (the ring sink emptying the flows from the jets) independent of the width of the ring, while as this ratio approaches infinity also a limiting value independent of $H$ is approached if any instability of the shear layers at the jet exits is suppressed. The very weak dependence on $H$ is a consequence of these two limiting values not differing much, with the strain rates for intermediate values of $H$ lying between them. Although flow-field aspects such as the development of Kelvin-Helmhotz instabilities in the outer shear layers also may be expected to occur in the presence of significant flow resistance at the duct exits, the virtual lack of dependence of the strain rate on $H$ will fail to apply, with it becoming inversely proportional to $H$ and independent of $R$ when $H / R$ approaches zero [22]. These new computational results help to clarify characteristics of axisymmetric open-duct and nozzle-flow types of laminar counterflows and may aid in considerations of experimental designs of such devices.

\section{Acknowledgements}

This work was supported by the Spanish MCINN through projects \# CSD2010-00010 and MTM2015-67030-P. FAW is supported by the US National Science Foundation through award \#CBET-1404026. 


\section{References}

[1] F.N. Egolfopoulos, N. Hansen, Y. Ju, K. Kohse-Hinghaus, C.K. Law, F. Qi, Advances and challenges in laminar flame experiments and implications for combustion chemistry, Prog. Ener. Combust. Sci. 43 (2014) 36-67.

[2] U. Niemann, K. Seshadri, F. A. Williams, Accuracies of laminar counterflow flame experiments, Combust. Flame 162 (2015) 1540-1549.

[3] M.L. Shay, P.D. Ronney, Nonpremixed edge flames in spatially varying straining flows. Combust. Flame 112 (1998) 171-180.

[4] J.S, Kim, P.A. Libby, F. A. Williams, On the displacement effects of laminar flames. Combust. Sci. Technol. 87 (1993) 1-25.

[5] M.D. Smooke, V. Giovangigli, Formulation of the premixed and nonpremixed test problems, in: M.D. Smooke (Ed.), Reduced Kinetic Mechanisms and Asymptotic Approximations for Methane-Air Flames, Springer-Verlag, Berlin, Germany (1991), pp. 1-28.

[6] Cosilab collection, version 2.0.7, www.rotexo.com, 2007.

[7] N. Peters, Turbulent combustion, Cambridge University Press, Cambridge, U.K., 2000.

[8] J.C. Rolon, D. Veynante, J.P. Martin, F. Durst, Counter jet stagnation flows. Exp. Fluids 11 (1991) 313-324.

[9] E. Korusoy, J.H. Whilelaw, Inviscid, laminar and turbulent opposed flows. Int. J. Numer. Meth. Fluids 46 (2004) 1069-1098.

[10] J.M. Bergthorson, K. Sone, T.W. Mattner, P.E. Dimotakis, D.G. Goodwin, D.I. Meiron, Impinging laminar jets at moderate Reynolds numbers and separation distances, Phys. Rev. E. 72 (2005) 066307.

[11] A. Liñán, D. Martínez-Ruiz, A.L. Sánchez, J. Urzay, Regimes of spray vaporization and combustion in counterflow configurations, Combust. Sci. Technol. 187 (2015) 103-131.

[12] W. Schach, Deflection of a circular fluid jet by a flat plate perpendicular to the flow direction, Ing. Arch. 6 (1935) 51-59.

[13] T. Strand, Inviscid-incompressible-flow theory of normal and slightly oblique impingement of a static round jet on the ground. J. Aircraft 4 (1967) 466-472.

[14] A. Rubel, Computations of jet impingement on a flat surface, AIAA J. 18 (1980) 168175.

[15] M.D. Deshpande, R.M. Vaishnav, Submerged laminar jet impingement on a plane, J. Fluid Mech. 114 (1982) 213-236.

[16] A. Rubel, Inviscid axisymmetric jet impingement with recirculating stagnation regions, AIAA J. 21 (1983) 351-357. 
[17] D.J. Phares, G.T. Smedley, R.C. Flagan, The inviscid impingement of a jet with arbitrary velocity profile, Phys. Fluids 12 (2000) 2046-2055.

[18] F.A. Williams, Combustion Theory, 2nd Ed., Addison-Wesley Publishing Co., Redwood City, CA, 1985.

[19] G.K. Batchelor, An Introduction to Fluid Dynamics, Cambridge University Press, Cambridge, 1967, pp. 507-509 and 536-538.

[20] W.-. Li, T.-L. Yao, H.-F. Liu, F.-C. Wang, Experimental investigation of flow regimes of axisymmetric and planar opposed jets, AIChE J. 57 (2011) 1434-1445.

[21] J. Carpio, J.L. Prieto, M. Vera, A local anisotropic adaptive algorithm for the solution of low-Mach transient combustion problems, J. Comput. Phys. 306 (2016) 19-42.

[22] K. Seshadri, F. A. Williams, Laminar flow between parallel plates with injection of a reactant at high Reynolds number, Int. J. Heat Mass Tran. 21 (1978) 251-253.

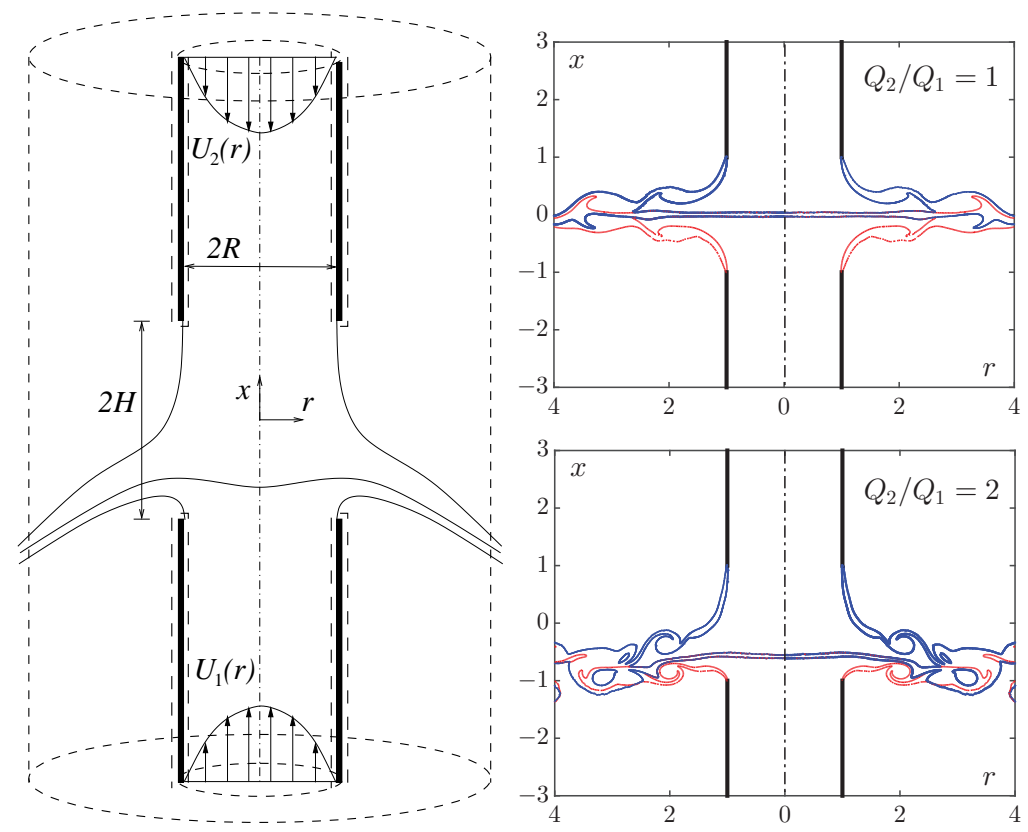

Figure 1: The left-hand-side plot shows a sketch of the counterflow configuration investigated here, including the separating stream surfaces and the boundaries of the integration domain. The right-hand-side plots show the isosurfaces of mass fractions $Y_{1}=(0.1,0.9)$ and $Y_{2}=(0.1,0.9)$ obtained with $R e=1000, H / R=1$, $\rho_{2} / \rho_{1}=1$, and $U_{1}=U_{2}=1$ for two different values of $Q_{2} / Q_{1}$. 
Figure 2: The upper plot shows dividing streamlines $x=x_{s}(r)$ obtained by integrating the Navier-Stokes equations for $H / R=1$ and $\rho_{2} / \rho_{1}=1$ with $R e=1000$ while the lower plots show the variation with $H / R$ of the location $x_{o}$ of the stagnation point and of the local curvature $1 / r_{c}$ of the dividing streamline there, also for $\rho_{2} / \rho_{1}=1$ and $R e=1000$.
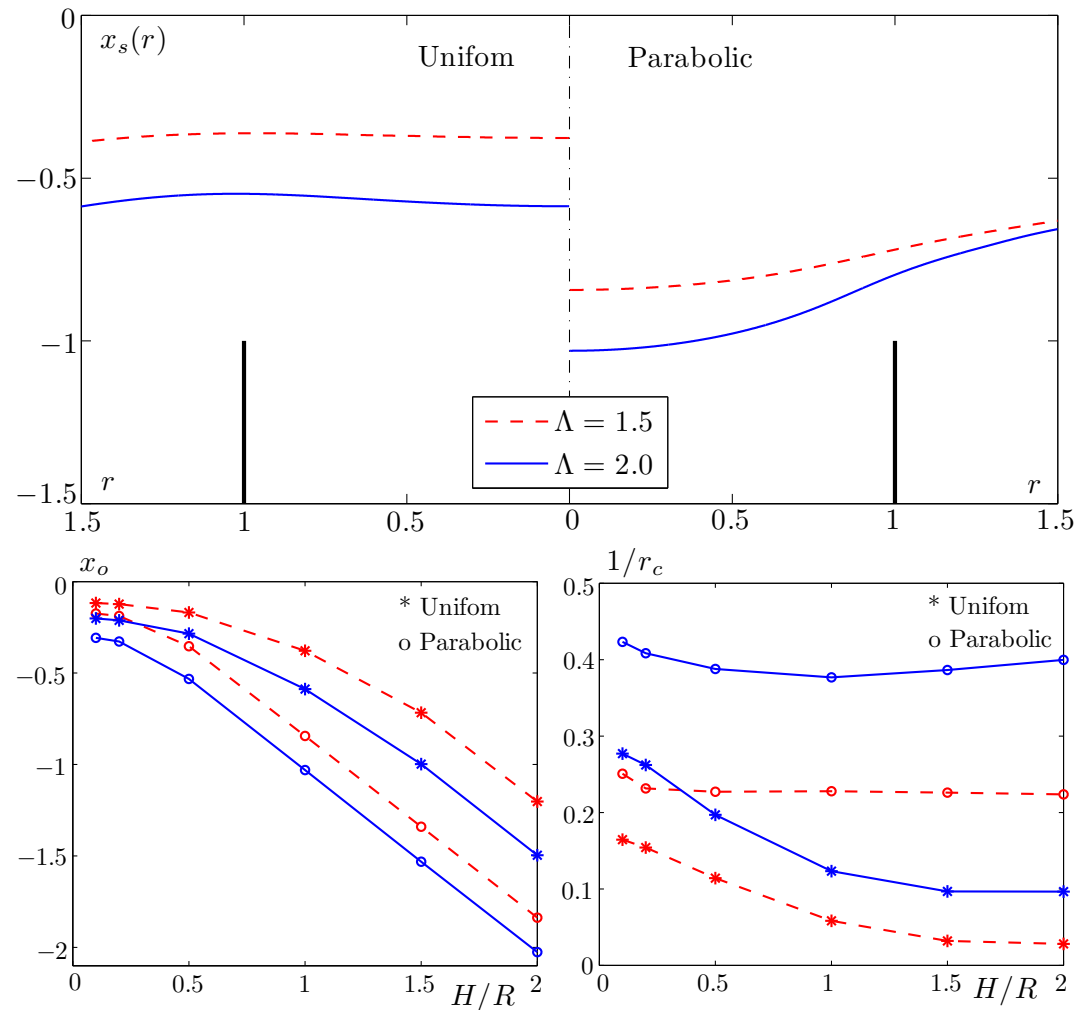

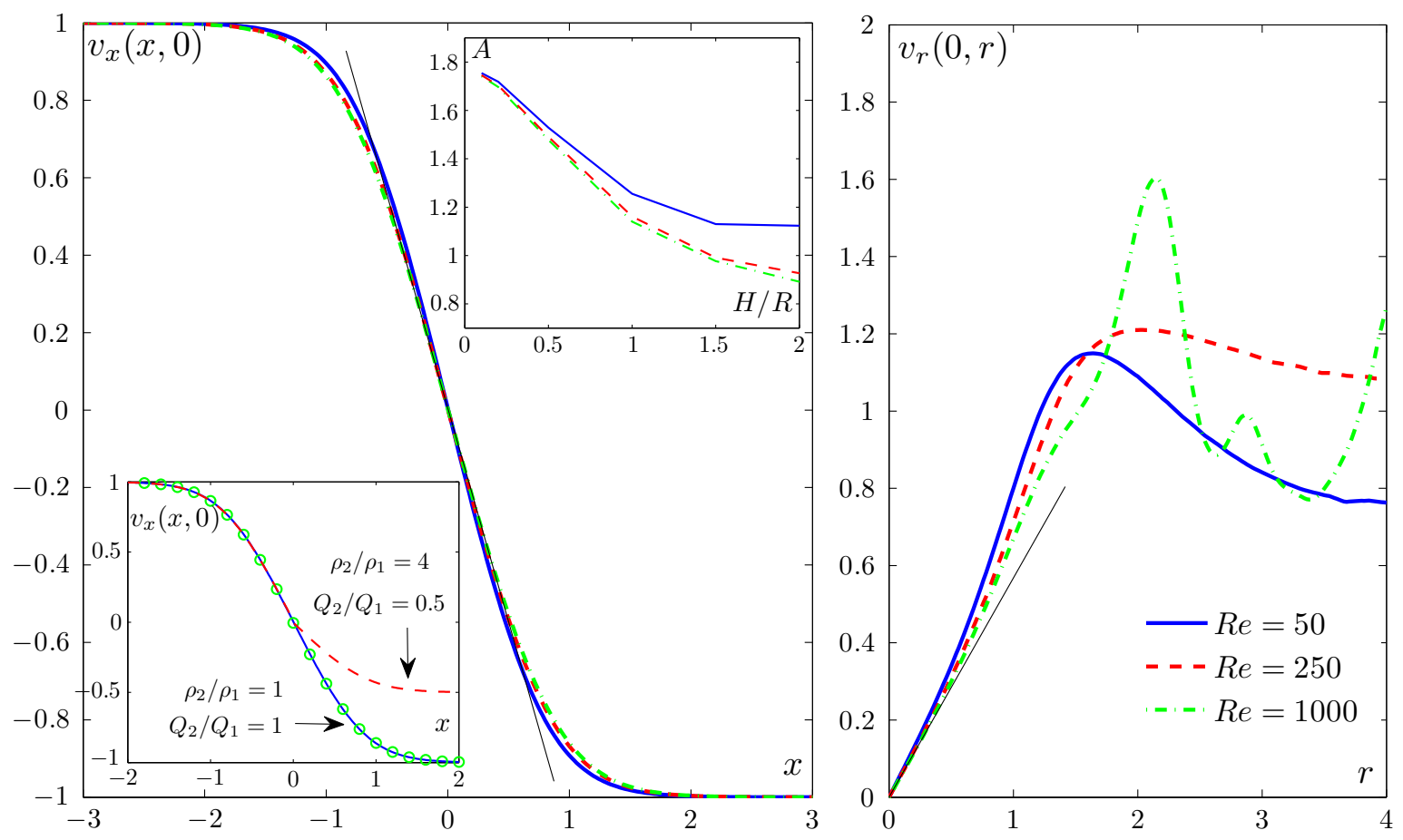

Figure 3: The axial velocity at $r=0$ in terms of $x$ and the radial velocity at $x=0$ in terms of $r$ obtained with $H / R=1, \rho_{2} / \rho_{1}=1, Q_{2} / Q_{1}=1$, and $U_{1}=U_{2}=1$ for three different values of $R e$ for uniform inlet flow. The upper inset shows the variation of the dimensionless strain rate $A$ with $H / R$, The lower inset compares the distribution of $v_{x}(x, 0)$ obtained for $H / R=1$ and $U_{1}=U_{2}=1$ with $\rho_{2} / \rho_{1}=1$ and $Q_{2} / Q_{1}=1$ (solid curve) and with $\rho_{2} / \rho_{1}=4$ and $Q_{2} / Q_{1}=0.5$ (dashed curve), with the circles denoting the density-weighted velocity $\rho^{1 / 2} v_{x}$.

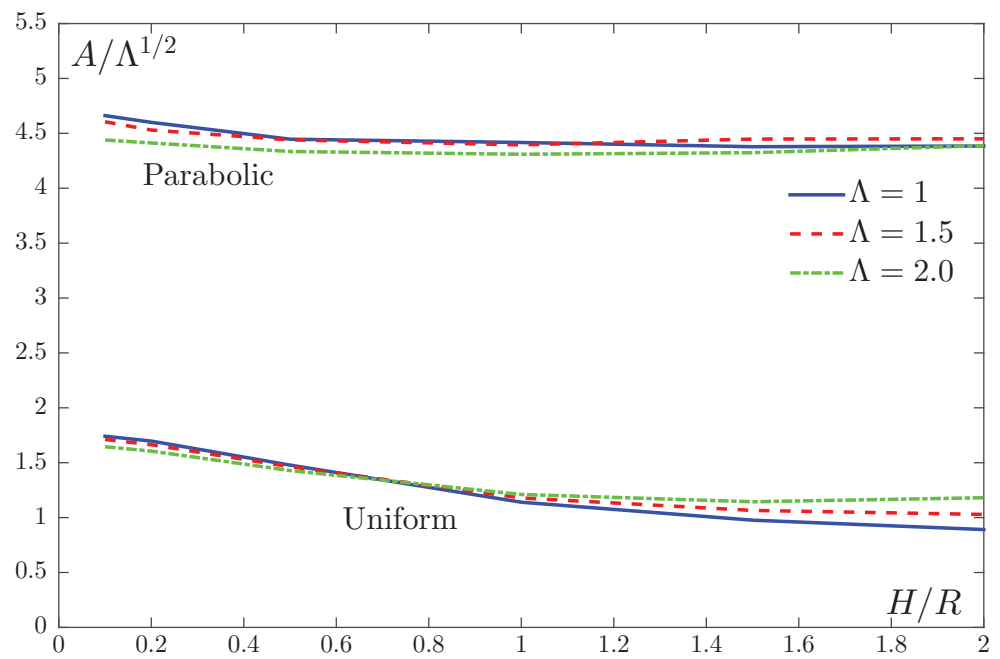

Figure 4: The variation of $A / \Lambda^{1 / 2}$ with $H / R$ for uniform and parabolic boundary velocities and three different values of $\Lambda$. 